\title{
Implications of Nanobiosensors in Agriculture
}

\author{
Vineeta Rai, Sefali Acharya, Nrisingha Dey ${ }^{*}$
}

Institute of Life Sciences, Division of Gene Function and Regulation, Laboratory of Plant Biotechnology, Bhubaneswar (Odisha), India.

Email: \{vineeta7885, sefaliacharya\}@gmail.com, *dey@ils.res.in, nrisinghad@yahoo.com

Received February $29^{\text {th }}, 2012$; revised March $28^{\text {th }}$, 2012; accepted April $12^{\text {th }}, 2012$

\begin{abstract}
Nanotechnology has emerged as a boon to the society with immense potential in varied area of research and our dayto-day life. The application of nanotechnology for the advancement of biosensor leads to an efficient nanobiosensor with miniature structure as compared to conventional biosensors. Nanobiosensors can be effectively used for sensing a wide variety of fertilizers, herbicide, pesticide, insecticide, pathogens, moisture, and soil pH. Taken together, proper and controlled use of nanobiosensor can support sustainable agriculture for enhancing crop productivity.
\end{abstract}

Keywords: Nanobiotechnology; Nanobiosensors; Sustainable Agriculture

\section{Introduction}

Accessibility of net land and water-resources for agriculture is rapidly declining, causing huge loss in agricultural output. Besides, the ever increasing concentration of herbicides, pesticides and heavy metals in agricultural land is alarming. These issues can only be dealt efficiently with the aid and continuous flow of new technologies into this sector. Presently, nanotechnology is visualized as a rapidly evolving field with high potential to revolutionize agricultural and food systems. It is viewed as a potential tool to enhance the quality of the agricultural based products and natural resource. It may boosts rural economy by promoting sustainable agriculture, facilitating farmcosts reduction and up lift product-values [1]. Considering the present scenario and merits of nanotechnology the paper reviews the implications of nanobiosensors in promoting agriculture to feed the growing populations. Nanobiosensors may be of great value for effective monitoring of soil quality in terms of its constituents, $\mathrm{pH}$, humidity, microbial load etc. and thus aid as an efficient tool to enhance productivity.

Nanobiosensor is a modified version of a biosensor which may be defined as a compact analytical device/ unit incorporating a biological or biologically derived sensitized element linked to a physico-chemical transducer [2]. In the year 1967, the first biosensor was invented [3] which led to the development of several modified biosensors. Interestingly, since early 20th century the concept of biosensors existed but their uses were limited only in laboratories and with advent of sciences several modern

*Corresponding author. biosensors were designed (Figure 1). Overall, there are three so-called "generations" of biosensors; first generation biosensors operates on electrical response, second generation biosensors functions involving specific "mediators" between the reaction and the transducer for generating improved response, and in third generation biosensors the reaction itself causes the response and no product or mediator diffusion is directly involved [4-18] (Figure 1).

\section{Nanobiosensors}

With the progression in sciences, nanobiosensors with superbly dedicated miniature sensors with highly miniaturization were designed and developed in 21st century based on the ideas of nanotechnology. Recently, researchers have used an integrated approach by combining nanosciences, electronics, computers and biology to create biosensors with extraordinary sensing capabilities that show unprecedented spatial and temporal resolution and reliability. Nanosensors with immobilized bioreceptor probes that are selective for target analyte molecules are called nanobiosensors. A nanobiosensor is usually built on the nanoscale to obtain process and analyze the data at the level of atomic scale (http://www.nanomedicine.com/NMI/Glossary.htm). Nanobiosensors open up new opportunities for basic research and provide tools for real bio-analytical applications, which was impossible in the past [19-22]. They can be integrated into other technologies such as lab-on-a-chip to facilitate molecular analysis. Their applications include detection of analytes like urea, glucose, pesticides etc., monitoring of metabolites and detection of various microorganisms/pathogens. 
- Report on the immobilization of proteins [4]

- First glass $\mathrm{pH}$ electrode [5]

- First paper on the oxygen electrode [6]

- First description of a biosensor [7]

- First potentiometricbiosensor [8]

- Invention of the Ion-Selective Field-Effect Transistor (ISFET) [9]

- The use of thermal transducers for biosensors was proposed [10]

- the term "optode" was coined to describea fibre-opticsensor [11]

- incorpcation of an electrochemical glucose biosensor in a bedside artificial pancreas [12]

- Karl Camman coined theterm biosensor [13]

- first needle-type enzyme eletrode was described [14]

- Paper on the use of ferrocene and its derivatives as an immobilised mediator for use with oxidoreductases [15]

- MediSense (Cambridge,USA)lauched a screen-printed enzyme electrodes for home blood-glucose monitoring

- Launch of the Pharmacia BIA Core SPR-based biosensor system

- i-STAT launcheshand-held blood analyzer

- Glucocard launched

- Launch of LifeScan Fast Take blood glucose biosensor

- Use of electrodeposition paints (EDPs) as immobilizationmatrices for biosensors [16]

- An enzymaticglucose $/ \mathrm{O}_{2}$ fuel cell was implanted in a living plant [17]

- An implanted glucose bilsensor operated for five days [18]

Figure 1. Milestone in the advancement of biosensors in $20^{\text {th }}$ century.

Their portability makes them ideal for their applications in field but they can be used in the laboratory setting as well.

\subsection{Characteristics for an Ideal Nanobiosensors}

- Highly specific for the purpose of the analyses i.e. a sensor must be able to distinguish between analyte and any "other" material.

- Stable under normal storage conditions.

- Specific interaction between analytes should be independent of any physical parameters such as stirring, $\mathrm{pH}$ and temperature.
- Reaction time should be minimal.

- The responses obtained should be accurate, precise, reproducible and linear over the useful analytical range and also be free from electrical noise.

- The nanobiosensor must be tiny, biocompatible, nontoxic and non-antigenic.

- Should be cheap, portable and capable of being used by semi-skilled operators.

\subsection{Constituents of Nanobiosensors}

A typical nanobiosensor comprises of 3 components; biologically sensitized elements (probe), transducer and 
detector [23] as described in Figure 2:

1) The biologically sensitized elements (probe) including receptors, enzymes, antibodies, nucleic acids, molecular imprints, lectins, tissue, microorganisms, organelles etc., which are either a biologically derived material or bio-mimic component that receives signals from the analytes (sample) of interest and transmits it to transducer. And such nano-receptor may play a vital role in the development of future nanobiosensors.

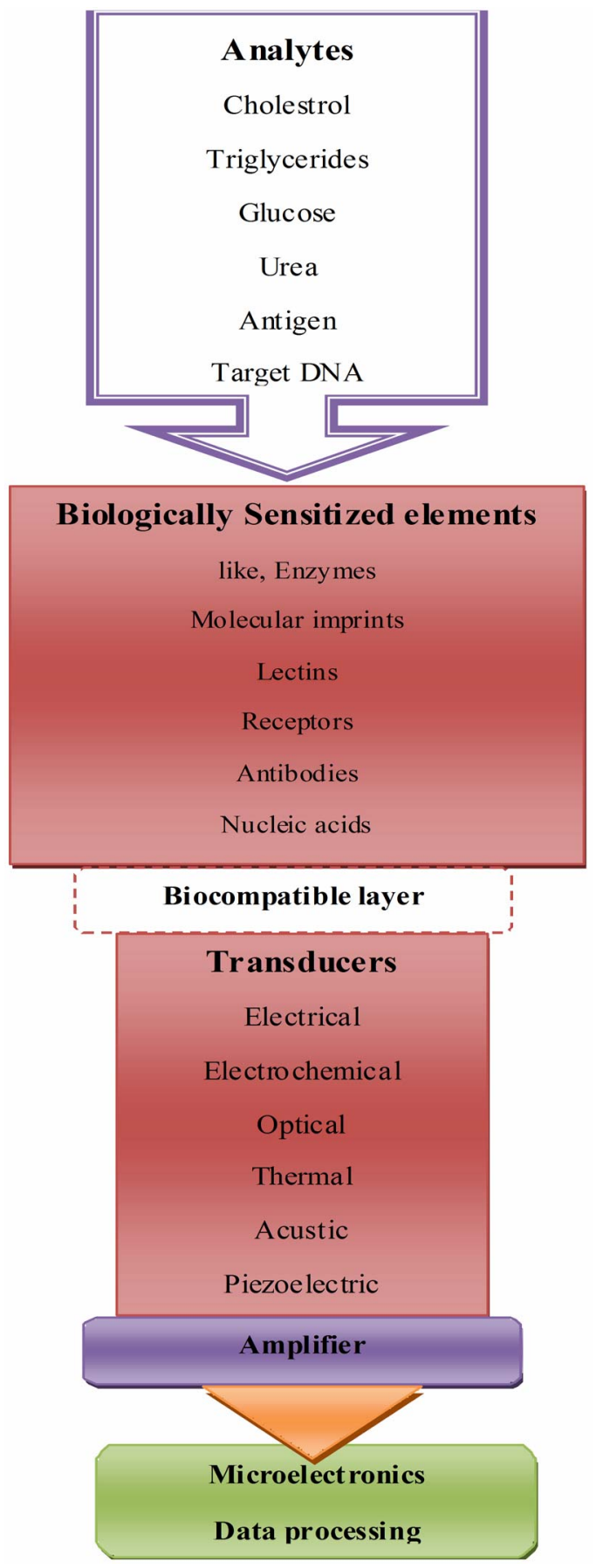

Figure 2. Principle of nanobiosensor.
2) The transducer acts as an interface, measuring the physical change that occurs with the reaction at the bioreceptor/sensitive biological element then transforming that energy into measurable electrical output. Depending on the mode of action transducers may be classified into following categories and discussed in details (Table 1).

3) The detector element traps the signals from the transducer, which are then passed to a microprocessor where they are amplified and analyzed; the data is then transferred to user friendly output and displayed/stored [24].

\subsection{Advantages of Nanobiosensors over Conventional Biosensors}

- These sensors are ultra sensitive and can detect single virus particles or even ultra-low concentrations of a substance that could be potentially harmful.

- Nanobiosensors works at atomic scale with highest efficiency.

- Nanobiosensors also have increased surface to volume ratio.

- 2.4. Disadvantages of nanobiosensors.

- Nanobiosensors are very sensitive and error prone.

- Nanobiosensors are still under infancy stage.

\section{Types of Nanobiosensors}

\subsection{Mechanical Nanobiosensors}

Nanoscale mechanical forces between biomolecules provide an exciting ground to measure the biomolecular interaction. This helps in the development of minute, sensitive and label free biosensors [25]. Microscale cantilever

Table 1. The mode of action transducers.

\begin{tabular}{|c|c|c|c|}
\hline $\begin{array}{l}\text { Sl. } \\
\text { No. }\end{array}$ & Transducer system & Principle & Applications \\
\hline 1. & Enzyme electrode & Amperometric & $\begin{array}{l}\text { Enzyme substrate and } \\
\text { immunological system }\end{array}$ \\
\hline 2. & Conductometer & Conductance & Enzyme substrate \\
\hline 3. & Piezoelectric crystal & Mass change & Volatile gases and vapors \\
\hline 4. & Thermistor & Calorimetric & $\begin{array}{l}\text { Enzyme, organelle, whole } \\
\text { cell or tissue sensors for } \\
\text { substrates, } \\
\text { Products, gases, pollutants, } \\
\text { antibiotics, vitamins, etc. }\end{array}$ \\
\hline 5. & $\begin{array}{l}\text { Optoelectronic/wave } \\
\text { guide and fiber optic } \\
\text { device }\end{array}$ & Optical pH & $\begin{array}{l}\text { enzyme substrates and } \\
\text { immunological systems }\end{array}$ \\
\hline 6. & $\begin{array}{l}\text { Ion sensitive } \\
\text { electrode (ISE) }\end{array}$ & Potentiometric & $\begin{array}{l}\text { Ions in biological media, } \\
\text { enzyme electrodes, enzyme } \\
\text { immunosensors }\end{array}$ \\
\hline 7. & $\begin{array}{l}\text { Field effect transistor } \\
\text { (FET) }\end{array}$ & Potentiometric & $\begin{array}{c}\text { Ions, gases, enzyme } \\
\text { substrates and immunological } \\
\text { analytes }\end{array}$ \\
\hline
\end{tabular}


(Figure 3) beams can be used to identify biomolecules by deflecting upon interaction with a specific biomolecule. By measuring the level of deflection each cantilever beam experiences in response to interactions with the molecules, the amount of analyte in the solution can be quantified and interpreted. Basically, there are three mechanisms to transduce the recognition of the analyte of interest into micromechanical bending of the cantilever, a) bending in response to a surface stress (Figure 4(a)); b) bending in response to a mass loading (Figure 4(b)); c) bending as a result of a temperature change (Figure 4(c)) [26]. The advantage of nano-mechanical devices is that they are highly mass sensitive. More the size decreases, more the mass reduces and hence the addition of bound analyte molecules results in an increased relative change to the main mass.

\subsection{Optical Nanobiosensors}

Optical biosensors are based on the arrangement of optics where beam of light is circulated in a closed path (Figure 4) and the change is recorded in resonant frequency when the analyte binds to the resonator. The resonator can be basically divided into linear resonator (light bounces between two end mirrors) and ring resonators (light is circulated in two different directions as end mirrors are absent). Unlike mechanical resonators (above mentioned) optical ones are based on the oscillating light within a cavity. Most of the commercially available optical biosensors rely on the use of lasers to monitor and quantify interactions of biomolecules that occur on specially derived surfaces or biochips [27]. Surface plasmon resonance (SPR) is an optical-electrical phenomenon involving the interaction of light with the electrons of a metal. It is based on the transfer of the energy carried by photons of light to a group of electrons (a plasmon) at the surface of a metal. Miniature optical sensors that specifically identify low concentrations of environmental and biological substances are in high demand. Recently, a triangular silver nanoparticle with remarkable optical properties and enhanced sensitivity to their nano environment has been developed [28].

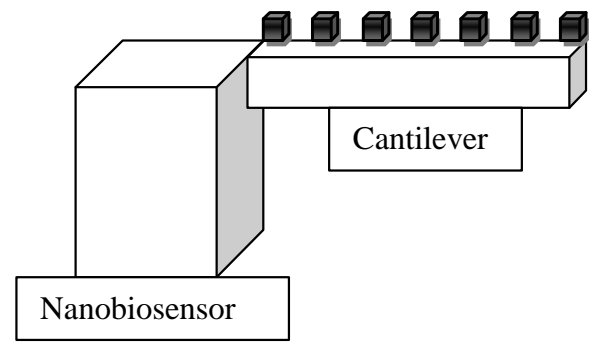

(a)

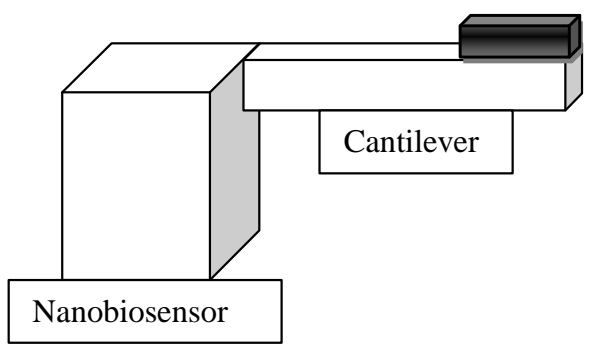

(b)

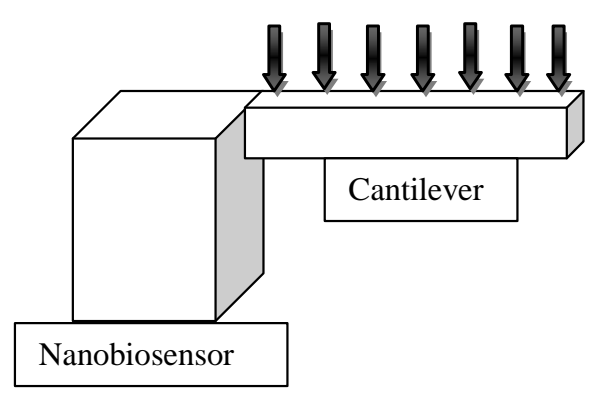

(c)

Figure 3. (a) Cantilever experiencing surface stress; (b) Cantilever experiencing mass load; (c) Cantilever experiencing temperature change.

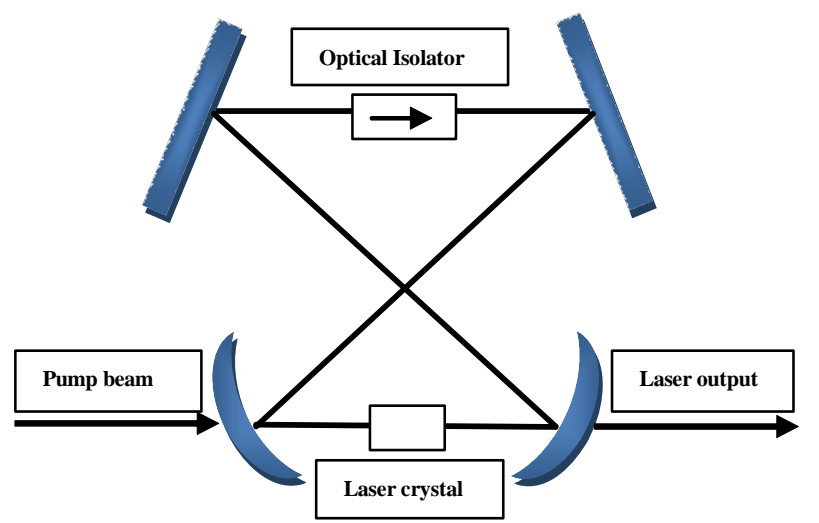

Figure 4. Optical biosensors based on the arrangement of mirrors. 


\subsection{Nanowire Biosensors}

Nanowire biosensor is a hybrid of two molecules that are extremely sensitive to outside signals: single stranded DNA, (serving as the "detector") and a carbon nanotube, (serving as the transmitter). The surface properties of nanowires can be easily modified using chemical or biological molecular ligands, which make them analyte independent [29]. This transduces the chemical binding event on their surface into a change in conductance of the nanowire with extreme sensitivity, real time and quantitative fashion. Boron-doped silicon nanowires (SiNWs) have been used to create highly sensitive, real-time electrically based sensors for biological and chemical species [30].

\subsection{Ion Channel Switch Biosensor Technologies}

The Ion Channel Switch (ICS) is based on a synthetic self-assembling membrane that acts as a biological switch for detecting the signals i.e. the presence of specific molecules by triggering an electrical current [31]. It delivers precise and quantitative test results in an immediate timeframe and reduces the time of emergency diagnoses from hours down to minutes.

\subsection{Electronic Nanobiosensors}

Electronic nanobiosensors work by electronically detecting the binding of a target DNA that actually forms a bridge between two electrically separated wires on a microchip [32]. Each chip contains multiple sensors, which can be independently addressed with capture probes for different target DNA molecules from the same or different organisms.

\subsection{Viral Nanobiosensors}

Virus particles are essentially biological nanoparticles. Herpes simplex virus (HSV) and adenovirus have been used to trigger the assembly of magnetic nanobeads as a nanosensor for clinically relevant viruses [33].

\subsection{PEBBLE Nanobiosensors}

Probes Encapsulated by Biologically Localized Embedding (PEBBLE) nanobiosensors consist of sensor molecules entrapped in a chemically inert matrix by a microemulsion polymerization process that produces spherical sensors in the size range of 20 to $200 \mathrm{~nm}$. Various sensor molecules can be entrapped including those that detect optical change [34], change in $\mathrm{pH}$ or $\mathrm{Ca}^{2+}$ ions [35] or can detect the fluorescence [36]. These nanosensors are capable of monitoring real-time inter- and intra-cellular imaging of ions and molecules, while at the same time they are also insensitive to interference from proteins and show great reversibility and stability to leaching and photobleaching. In human plasma they demonstrate a robust oxygen sensing capability, little affected by light scattering and autofluorescence [37].

\subsection{Nanoshell Biosensors}

Positioning gold nanoshells are used in a rapid immunoassay for detecting analytes within complex biological media without any sample preparation [38]. Aggregation of antibody/nanoshell conjugates with extinction spectra in the near infrared is monitored spectroscopically in the presence of analyte. Nanoshells can enhance chemical sensing by as much as 10 billion times [32].

\section{Role of Nanobiosensor in Agriculture}

Presently, nanomaterial-based biosensors exhibit fascinating prospects over traditional biosensors. Nanobiosensors have marked advantages such as enhanced detection sensitivity/specificity and possess great potential for its applications in different fields including environmental and bioprocess control, quality control of food, agriculture, bio defence, and, particularly, medical applications. But here we are concerned with the role of nano biosensor in agriculture and agro-products. Some of the potential applications of nanobiosensors are listed below:

\subsection{As Diagnostic Tool for Soil Quality and Disease Assessment}

Nano sensors may be used to diagnose soil disease (caused by infecting soil micro-organisms, such as viruses, bacteria, and fungi) via the quantitative measurement of differential oxygen consumption in the respiration (relative activity) of "good microbes" and "bad microbes" in the soil. The measurement proceeds through the following steps: two sensors impregnated with "good microbes" and "bad microbes" respectively, are immersed in a suspension of soil sample in buffer solution and the oxygen consumption data by two microbes were detected. By comparing two data, we can easily decide which microbe favors the soil. Apart from that, we can also predict whether or not soil disease is ready to break out in the tested soil beforehand. So, it is to be emphasized that the biosensor offers an innovative technique of diagnosing soil condition based on semi-quantitative approach [39].

\subsection{As an Agent to Promote Sustainable Agriculture}

A nanofertilizer refers to a product that delivers nutrients to crops encapsulated within a nanoparticle. There are three ways of encapsulation: a) The nutrient can be encapsulated inside nanomaterials such as nanotubes or nanoporous materials; b) coated with a thin protective 
polymer film; c) delivered as particles or emulsions of nanoscale dimensions. Nanofertilizers could be used to reduce nitrogen loss due to leaching, emissions, and long-term assimilation by soil microorganisms [40]. Recently carbon nanotubes were shown to penetrate tomato seeds [41], and zinc oxide nanoparticles were shown to enter the root tissue of ryegrass [42]. This suggests that new nutrient delivery systems that exploit the nanoscale porous domains on plant surfaces can be developed. But, the nanofertilizers should show sustained release of nutrients on-demand while preventing them from prematurely converting into chemical/gaseous forms that can not be absorbed by plants. To achieve this, biosensor could be attached to this nanofertilizer that allows selective nitrogen release linked to time, environmental and soil nutrient condition. Slow-controlled-release of fertileizers may also improve soil by decreasing toxic effects associated with fertilizer over application.

Zeolites are naturally occurring crystalline aluminum silicates that can a) enable better plant growth; b) improve the efficiency and value of fertilizer; c) improve water infiltration and retention; d) improves yield; e) retain nutrients for use by plants; f) improve long term soil quality and g) reduce loss of nutrients in soil. Zeolite holds nutrients in the root zone for plants to use when required. This leads to more efficient use of $\mathrm{N}$ and $\mathrm{K}$ fertilisers-either less fertiliser for the same yield or the same amount of fertiliser lasting longer and producing higher yields. An added benefit of zeolite application is that unlike other soil amendments (gypsum and lime) it does not break down over time but remains in the soil to help improve nutrient and water retention permanently. With subsequent applications, the zeolite will further improve the soil's ability to retain nutrients and produce improved yields. Zeolites linked to a nanobiosensor can modernize agriculture in the sense that the biosensor can sense the deficiency in either plant or soil and control the release of water/nutrients retained in the zeolite.

Pesticides inside nanoparticles are being developed that can be timed-release or have release linked to an environmental trigger [43]. Also, combined with a smart delivery system, herbicide could be applied only when necessary, resulting in greater production of crops and less injury to agricultural workers.

\subsection{As a Device to Detect Contaminants and Other Molecules}

Several nanobiosensors are designed to detect contaminants, pests, nutrient content, and plant stress due to drought, temperature, or pressure. They may also potentially helpful for farmers to enhance competence by applying inputs only when necessary. Organophosphorus pesticides such as dichlorvos and paraoxon, at very low levels could be monitored by liposome-based biosensors
[44]. Zhang et al. [45] developed a method for the detection of Escherichia coli (E. coli) using bismuth nanofilm modified GCE based on the principle of flow injection analysis (FIA). Seo et al. [46] constructed a biochip sensor system, consisting of two Ti contact pads and a 150 $\mathrm{nm}$ wide Ti nanowell device on $\mathrm{LiNbO}_{3}$ substrate. When the bacteria were resistant to the phages (uninfected bacteria), small voltage fluctuations were observed in the nanowell displaying a power spectral density (PSD). The biosensors developed using PSII (photosystem II), known to bind several groups of herbicides, isolated from photosynthetic organisms may have potential to monitor polluting chemicals, leading to the set-up of a low cost, easy-to-use apparatus, able to reveal specific herbicides, and eventually, a wide range of organic compounds present in industrial and urban effluents, sewage sludge, landfill leak-water, ground water, and irrigation water [47].

\subsection{As Tool for Effective Detection of DNA and Protein}

There are several nanosensors like ssDNA-CNTs probes/ optical biosensors to detect specific kinds of DNA oligonucleotides [48], MWNTs/ZnO/CHIT composite film modified GCE for immobilization ssDNA probes to effectively discriminate different DNA sequences [49,50], a nanobiosensor with bionanocomposite layer of MWNT in chitosan deposited on a SPCE for the detection of deep DNA damage [51], a nanobiosensor with GNPs functionalized with alkanethiol-capped LNA/DNA chimeras in a tail-to-tail hybridization mode for single-stranded DNA [52], Nano-SiO $2 /$ p-aminothiophenol (PATP) film for the detection of the PAT gene sequences by a labelfree EIS method [53]. Maki et al. [54] reported the first nanowire field effect transistor based biosensor which achieves simple and ultra-sensitive electronic DNA methylation detection and avoids complicated bisulfite treatment and PCR amplification.

Similarly, using protein-ligand (antigen) interaction properties, protein-nanoparticles based biosensors can realize the ultra-sensitive detection of special protein molecules. The use of these DNA and protein detecting biosensors might play a vital role in detection of plant pathogens; certain abnormalities in plants linked to mineral deficiency, biomarkers, and discriminate one plant species from another etc.

\subsection{As a Tool for Analysis in Food Products}

Biosensor-based analysis is becoming increasingly important in the food industry where it has several applications;

- Vitamins analysis: The SPR biosensor monitors interactions of a specific binding protein with the vita- 
min immobilized on a CM5 sensor chip.

- Antibiotics detection: Recently the presence of prohibited antibiotics was detected in honey. Biosensors analyze the presence of antibiotics reliably, effecttively and in a short time.

- Detection of food spoilage: Amperometric biosensor using immobilized enzyme diamine oxidase (DAO) has been developed for the rapid monitoring of the histamine levels in tiger prawn (Penaeus monodon), similarly a potentiometric biosensor could analyse isocitrate using a $\mathrm{CO}_{3}^{2-}$-selective electrode and enzyme immobilization in flow injection analysis (FIA)

- Detection of microbial contamination: Immunobiosensors based on the surface immobilization of monoclone antibodies onto indium tin oxide (ITO) electrodes could detect Escherichia coli O157:H7.

\section{Market Assessment, Risks, Regulation and Acceptance}

Biological systems are used to nano-scale materials like proteins, carbohydrates, fats etc., but the use on engineered nanomaterials in agriculture and edibles has raised concerns regarding the latent risks coupled with the widespread use of engineered nanomaterials for environment or human health or both. The major apprehensions being the insufficient knowledge about the factors like toxicity, bioaccumulation, and exposure risk associated with the use of nanobiosensors. Also, the present scenario is due to minimal funding for risk assessments in a research projects. Conducting reproducible and reliable biocompatibility studies with nanostructures is difficult by the tentative behavior of particulate matter in biological settings and the complicatedness in making in situ measurements of properties such as size, shape and surface chemistry. Due to the complexities the risk assessments should include an early valuation of projected sensors in order to recognize and address nano-bio interactions that may impact the development and commercialization of Center technologies. Expertise should be accessible during the testing of more mature prototypes to ensure safety and effectiveness of the sensor technology. The nano particles and the nanoscale materials used in the construction of nano biosensors has to be properly characterised and tested in biological environments and the probable toxicity has to be examined [55]. The possible hazard of nanoparticles to biological organisms has significantly drawn interest from academics, industry, governmental ethical committees and non-governmental organizations worldwide.

Besides, the commercialization of nanobiosensors is also linked to several risk factors [1] as: a) Initial applications "could act as substitutes for agricultural commodities" [1] which might be "disastrous" on the economy of developing countries [1,56]; b) Secondly, vari- able import regulations in different nations could obstruct the nano-product expansion [1,57]; c) Lastly, the use of nanotechnology could pose "negative economic effects on the poor by increasing productivity in developed countries" [1], which could lead to decrease commodity price in developing countries [1].

Apart from all the associated risks marketing of any product depends on the public acceptance of the same. Thus, risk perceptions are vital for the future accessibility of nano products worldwide [1]. Till date, consumers lean to be more unwilling to nanofood applications than other nanotechnology uses $[1,58]$ apparent "benefits and health risks affect acceptance" [1], "meaning consumers do not perceive all products with the same risk levels" [1,59] Risk communication strategies should articulate "attention to the messenger and the target of the message" [1,60]. Some "external factors" [1] could also put an impact in "shaping future acceptance in the key markets" [1] that will largely influence the future of nanotechnologies globally [1]. Non-governmental organizations which support a "ban on nanotechnology use in food and agriculture" may prove significant [1], approaching toward condensed commercialization internationally [1,61]. This might result in "technology divide" $[1,62]$.

The probable solution to current problem is the proper labeling on nano-products in certain developed countries which might lead to technology consumption and regulations in developing countries. The Science and Technology Committee of the UK House of Lords has recommended including a compulsory "pre-commercialization assessment using the methods supported by a research investment effort in risk assessment and detection methods" [1,63]. "The European Food Safety Agency supports the use of conventional risk assessment while addressing the limited knowledge on exposure to nanofood applications" $[1,64]$. While creating regulations countries should keep in mind the existing institutional capacity [1]. "Similarities between biotech regulatory systems and nanotech regulatory systems" [1] should be taken as an advantage [1] (Niosi and Reid, "Biotechnology and Nanotechnology"). Keeping in mind the learnt lessons "from the challenges observed in biosafety issues of biotech regulatory system" [1], the "need of public education, transparency and predictability" [1] has increased [1,65]. "The lack of risk assessment capacity" [1] and permeable borders should also support countries to form local groups [1].

\section{Future Perspectives}

Clearly, there is an opportunity for nanotechnology to have a profound impact on energy, the economy and the environment, by improving the screening processes. New prospects for integrating nanotechnologies into nanobio- 
sensors should be explored, cognizant of any potential risk to the environment or to human health. With targeted efforts by governments and academics in developing such enabled agro-products, we believe that nanotechnology will be transformative in the field of agriculture by focused research and development toward the goals for reaching sustainable agriculture.

\section{REFERENCES}

[1] G. Gruère, C. Narrod and L. Abbott, “Agriculture, Food, and Water Nanotechnologies for the Poor: Opportunities and Constraints," IFPRI Policy Brief, Vol. 19, 2011.

[2] A. P. Turner, "Biosensors-Sense and Sensitivity," Science, Vol. 290, No. 5495, 2000, pp. 1315-1317. doi:10.1126/science.290.5495.1315

[3] S. J. Updike and G. P. Hicks, "The Enzyme Electrode," Nature, Vol. 214, 1967, pp. 986-988. doi:10.1038/214986a0

[4] J. M. Nelson and E. G. Griffin, “Adsorption of Invertase,” Journal of the American Chemical Society, Vol. 38, No. 5, 1916, pp. 1109-1115. doi:10.1021/ja02262a018

[5] W. S. Hughes, "The Potential Difference between Glass and Electrolytes in Contact with the Glass," Journal of the American Chemical Society, Vol. 44, No. 12, 1922, pp. 2860-2867. doi:10.1021/ja01433a021

[6] L. C. Clark, "Monitor and Control of Blood and Tissue Oxygen Tensions," Transactions of the American Society for Artificial Internal Organs, Vol. 2, 1956, pp. 41-48.

[7] L. C. Clark Jr. and C. Lyons, "Electrode System for Continuous Monitoring in Cardiovascular Surgery,” Annals of the New York of Academy of Science, Vol. 148, No. 1962, pp. 133-153. doi:10.1111/j.1749-6632.1968.tb20346.x

[8] G. G. Guilbault and J. G. Montalvo, "A Urea-Specific Enzyme Electrode,” Journal of the American Chemical Society, Vol. 91, No. 8, 1969, pp. 2164-2165. doi:10.1021/ja01036a083

[9] P. Bergveld, "Development of an Ion-Sensitive SolidState Device for Neurophysiological Measurements," IEEE Transactions on Biomedical Engineering, Vol. BM17, No. 1, 1970, pp. 70-71. doi:10.1109/TBME.1970.4502688

[10] K. Mosbach and B. Danielsson, "An Enzyme Thermistor," Biochimica et Biophysica Acta (BBA)-Enzymology, Vol. 364, No. 1, 1974, pp. 140-145.

[11] D. W. Lubbers and N. Opitz, "The $\mathrm{pCO}_{2} / \mathrm{pO}_{2}$ Optrode: A New $\mathrm{pCO}_{2}, \mathrm{pO}_{2}$ Device for the Measurement of $\mathrm{pCO}_{2}$ or $\mathrm{pO}_{2}$ in Gases or Fluids,” Zeitschrift für Naturforschung C. A Journal of Biosciences, Vol. 30c, 1975, pp. 532-533.

[12] A. H. Clemens, P. H. Chang and R. W. Myers, "Development of an Automatic System of Insulin Infusion Controlled by Blood Sugar, Its System for the Determination of Glucose and Control Algorithms," Proceeedings Journees Annuelles de Diabtologie de l'Htel-Dieu, 1976, pp. 269-278.

[13] K. Cammann, "Biosensors Based on Ion-Selective Electrodes,” Fresenius Zeitschrift für Analytische Chemie,
Vol. 287, No. 1, 1977, pp. 1-9. doi:10.1007/BF00539519

[14] M. Shichiri, R. Kawamori, R.Yamaski, Y. Hakai and H. Abe, "Wearable Artificial Endocrine Pancreas with Needle-Type Glucose Sensor,” Lancet, Vol. 2, No. 8308, 1982, pp. 1129-1131. doi:10.1016/S0140-6736(82)92788-X

[15] A. E. G. Cass, D. G. Francis, H. A. O. Hill, W. J. Aston, I. J. Higgins, E. V. Plotkin, L. D. L. Scott and A. P. F. Turner, "Ferrocene-Mediated Enzyme Electrode for Amperometric Determination of Glucose," Analytical Chemistry, Vol. 56, No. 4, 1984, pp. 667-671. doi:10.1021/ac00268a018

[16] C. Kurzawa, A. Hengstenberg and W. Schuhmann, “Immobilization Method for the Preparation of Biosensors Based on pH Shift-Induced Deposition of BiomoleculeContaining Polymer Films,” Analytical Chemistry, Vol. 74, No. 2, 2002, pp. 355-361. doi:10.1021/ac010830a

[17] N. Mano, F. Mao and A. Heller, "Characteristics of a Miniature Compartment-Less Glucose- $\mathrm{O}_{2}$ Biofuel Cell and Its Operation in a Living Plant," Journal of the American Chemical Society, Vol. 125, No. 21, 2003, pp. 6588-6594. doi:10.1021/ja0346328

[18] R. L. Weinstein, J. R. Bugler, S. L. Schwartz, T. A. Peyser, R. L. Brazg and G. V. McGarraugh, "Accuracy of the 5-Day Freestyle Navigator Continuous Glucose Monitoring System-Comparison with Frequent Laboratory Reference Measurements,” Diabetes Care, Vol. 30, No. 5, 2007, pp. 1125-1130. doi:10.2337/dc06-1602

[19] C. You, M. Bhagawati, A. Brecht and J. Piehler, “Affinity Capturing for Targeting Proteins into Micro and NANOSTRUCTURES," Analytical and Bioanalytical Chemistry, Vol. 393, No. 6-7, 2009, pp. 1563-1570. doi:10.1007/s00216-008-2595-6

[20] M. N. Velasco, "Optical Biosensors for Probing at the Cellular Level: A Review of Recent Progress and Future Prospects,” Seminars in Cell \& Developmental Biology, Vol. 20, No. 1, 2009, pp. 27-33. doi:10.1016/j.semcdb.2009.01.013

[21] X. Fan, I. M. White, S. I. Shopova, H. Zhu, J. D. Suter and Y. Sun, "Sensitive Optical Biosensors for Unlabeled Targets: A Review," Analytica Chimica Acta, Vol. 620, No. 1-2, 2008, pp. 8-26. doi:10.1016/j.aca.2008.05.022

[22] V. K. Khanna, "New-Generation Nano-Engineered Biosensors, Enabling Nanotechnologies and Nanomaterials," Sensor Review, Vol. 28, No. 1, 2008, pp. 39-45. doi:10.1108/02602280810850017

[23] A. Shana and K. R. Rogers, "Biosensors," Measurement Science and Technology, Vol. 5, No. 5, 1994, pp. 461-472. doi:10.1088/0957-0233/5/5/001

[24] A. Cavalcanti, B. Shirinzadeh, M. Zhang and L. C. Kretly, "Nanorobot Hardware Architecture for Medical Defense," Sensors, Vol. 8, No. 5, 2008, pp. 2932-2958. doi:10.3390/s8052932

[25] M. M. Cheng, "Nanotechnologies for Biomolecular Detection and Medical Diagnostics,” Current Opinion in Chemical Biology, Vol. 19, 2006, pp. 10-11.

[26] C. Ziegler, "Cantilever-Based Biosensors," Analytical and Bioanalytical Chemistry, Vol. 379, No. 7-8, 2004, pp. 
946-959.

[27] T. Vo-Dinh, "Optical Nanosensors for Detecting Proteins and Biomarkers in Individual Living Cells," Methods in Molecular Biology, Vol. 300, 2005, pp. 383-402.

[28] A. J. Haes and R. P. Duyne, "Preliminary Studies and Potential Applications of Localized Surface Plasmon Resonance Spectroscopy in Medical Diagnostics,” Expert Review of Molecular Diagnostics, Vol. 4, No. 4, 2004, pp. 527-537. doi:10.1586/14737159.4.4.527

[29] http://seminarprojects.com/Thread-nano-sensors-and-dete ctors-their-applications-full-report\#ixzz1nYqLIR14

[30] Y. Cui, Q. Wei, H. Park and C. M. Lieber, “Nanowire Nanosensors for Highly Sensitive and Selective Detection of Biological and Chemical Species,” Science, Vol. 293, No. 12, 2001, pp. 89-92.

[31] B. A. Cornell, "Optical Biosensors: Present and Future," In: F. Lighler and C. R. Taitt, Eds., Membrane Based Biosensors, Chapter 457, Elsevier, Amsterdam, 2002, p. 12.

[32] K. K. Jain, "Nanotechnology in clinical Laboratory Diagnostics,” Clinica Chimica Acta, Vol. 358, No. 1-2, 2005, pp. 37-54. doi:10.1016/j.cccn.2005.03.014

[33] J. M. Perez, F. J. Simeone, Y. Saeki, L. Josephson and R. Weissleder, "Viral-Induced Self-Assembly of Magnetic Nanoparticles Allows the Detection of Viral Particles in Biological Media," Journal of the American Chemical Society, Vol. 125, No. 34, 2003, pp. 10192-10193. doi:10.1021/ja036409g

[34] H. A. Clark, "Optical Nanosensors for Chemical Analysis Inside Single Living Cells, 1: Fabrication, Characterization, and Methods for Intracellular Delivery of PEEBLE Sensors,” Analytical Chemistry, Vol. 71, No. 21, 1999, pp. 4831-4836. doi:10.1021/ac990629o

[35] H. A. Clark, "Optical Nanosensors for Chemical Analysis inside Single Living Cells, 2: Sensors for $\mathrm{pH}$ and Calcium and the Intracellular Application of PEEBLE Sensors," Analytical Chemistry, Vol. 71, No. 21, 1999, pp. 48374843. doi:10.1021/ac990630n

[36] J. P. Sumner, J. W. Aylott, E. Monson and R. Kopelman, "A Fluorescent PEBBLE Nanosensor for Intracellular Free Zinc,” Analyst, Vol. 127, 2002, pp. 11-16. doi:10.1039/b108568a

[37] Y. Cao, Y.E. Lee Koo and R. Kopelman, "Poly(decyl methacrylate)-Based Fluorescent PEBBLE Swarm Nanosensors for Measuring Dissolved Oxygen in Biosamples," Analyst, Vol. 129, No. 7, 2004, pp. 45-50.

[38] L. R. Hirsch, J. B. Jackson, A. Lee, N. J. Halas and J. L. West, "A Whole Blood Immunoassay Using Gold Nanoshells," Analytical Chemistry, Vol.75, No. 23, 2003, pp. 77-81.

[39] http://www.aist.go.jp/aiste/latestresearch/2004/20040402 _1/20040402_1.html

[40] M. C. DeRosa, C. Monreal, M. Schnitzer, R. Walsh and Y. Sultan, "Nanotechnology in Fertilizers," Nature Nanotechnology, Vol. 5, 2010, p. 91. doi:10.1038/nnano.2010.2

[41] M. Khodakovskaya, E. Dervishi, M. Mahmood, Y. Xu, Z.
Li, F. Watanabe and A. S. Biris, “Carbon Nanotubes Are Able to Penetrate Plant Seed Coat and Dramatically Affect Seed Germination and Plant Growth,” ACS Nano, Vol. 3, No. 10, 2009, pp. 3221-3227. doi:10.1021/nn900887m

[42] D. H. Lin and B. S. Xing, "Root Uptake and Phytotoxicity of ZnO Nanoparticles,” Environmental Science \& Technology, Vol. 42, No. 15, 2008, pp. 5580-5585. doi:10.1021/es800422x

[43] C. Lauderwasser, "Small Sizes That Matter: Opportunities and Risks of Nanotechnologies,” Report in Cooperation with the OECD International Futures Programme. http://www.oecd.org/dataoecd/32/1/44108334.pdf

[44] V. Vamvakaki and N. A. Chaniotakis, "Pesticide Detection with a Liposome-Based Nano-Biosensor," Biosensors and Bioelectronics, Vol. 22, No. 12, 2007, pp. 28482853. doi:10.1016/j.bios.2006.11.024

[45] W. Zhang, H. Tang, P. Geng, Q. Wang, L. Jin and Z. Wu, "Amperometric Method for Rapid Detection of Escherichia coli by Flow Injection Analysis Using a Bismuth Nano-Film Modified Glassy Carbon Electrode," Electrochemistry Communications, Vol. 9, No. 4, 2007, pp. 833-838. doi:10.1016/j.elecom.2006.11.019

[46] S. Seo, M. Dobozi-King, R. F. Young, L. B. Kish and M. Cheng, "Patterning a Nanowell Sensor Biochip for Specific and Rapid Detection of Bacteria," Microelectronic Engineering, Vol. 85, No. 7, 2008, pp. 1484-1489. doi:10.1016/j.mee.2007.12.046

[47] M. T. Giardi and E. V. Piletska, "Biotechnological Applications of Photosynthetic Proteins: Biochips, Biosensors and Biodevices,” Biotechnology Intelligence Unit Copublished by Land Biosciences and Springer, 2006.

[48] C. Cao, J. H. Kim, D. Yoon, E. S. Hwang, Y. J. Kim and S. Baik, "Optical Detection of DNA Hybridization Using Absorption Spectra of Single-Walled Carbon Nanotubes," Materials Chemistry and Physics, Vol. 112, No. 3, 2008, pp. 738-741. doi:10.1016/j.matchemphys.2008.07.129

[49] W. Zhang, T. Yang, D. Huang, K. Jiao and G. Li, "Synergistic Effects of Nano-ZnO/Multi-Walled Carbon Nanotubes/Chitosan Nanocomposite Membrane for the Sensitive Detection of Sequence Specific of PAT Gene and PCR Amplification of NOS Gene,” Journal of Membrane Science, Vol. 325, 2008, pp. 245-251. doi:10.1016/j.memsci.2008.07.038

[50] W. Zhang, T. Yang, D. M. Huang and K. Jiao, “Electrochemical Sensing of DNA Immobilizationand Hybridization Based on Carbon Nanotubes/Nano Zinc Oxide/ Chitosan Composite Film," Chinese Chemical Letters, Vol. 19, 2008, pp. 589-591. doi:10.1016/j.cclet.2008.03.012

[51] J. Galandova, G. Ziyatdinova and J. Labuda, "Disposable Electrochemical Biosensor with Multiwalled Carbon Nanotubes-Chitosan Composite Layer for the Detection of Deep DNA Damage,” Analytical Science, Vol. 24, No. 6, 2008, pp. 711-716. doi:10.2116/analsci.24.711

[52] F. McKenzie, K. Faulds and D. Graham, "SequenceSpecific DNA Detection Using High-Affinity LNAFunctionalized Gold Nanoparticles,” Small, Vol. 3, 2007, pp. 1866-1868. doi:10.1002/smll.200700225 
[53] Y. Ma, K. Jiao, T. Yang and D. Sun, "Sensitive PAT Gene Sequence Detection by $\mathrm{Nano}-\mathrm{SiO}_{2} / \mathrm{Paminothio-}$ phenol Self-Assembled Films DNA Electrochemical Biosensor Based on Impedance Measurement," Sensors and Actuators B, Vol. 131, No. 2, 2008, pp. 565-571. doi:10.1016/j.snb.2007.12.046

[54] W. C. Maki, N. N. Mishra, E. G. Cameron, B. Filanoski, S. K. Rastogi and G. K. Maki, "Nanowiretransistor Based Ultra-Sensitive DNA Methylation Detection,” Biosensor and Bioelectronics, Vol. 23, No. 6, 2008, pp. 780-787. doi:10.1016/j.bios.2007.08.017

[55] http://cnbs.centers.ufl.edu/research/sensors.asp

[56] ETC Group, “The Potential Impacts of Nano-Scale Technologies,” Meridian Institute, Nanotechnology, Commodities, and Development.

[57] G. Gruère and D. Sengupta, “GM-Free Private Standards and Their Effects on Biosafety Decision-Making in Developing Countries,” Food Policy, Vol. 34, No. 5, 2009, pp. 399-406. doi:10.1016/j.foodpol.2009.04.002

[58] B. Lyndhurst, “An Evidence Review of Public Attitudes to Emerging Food Technologies, Social Science Research Unit,” Food Standards Agency, Vol. 89, 2010. http://www.foodbase.org.uk/admintools/reportdocuments/ 3701647_Emerging_Food_Technologies_Final_Report_ with_logo.pdf

[59] M. Siegrist, N. Stampfli and H. Kastenholz, “Acceptance of Nanotechnology Foods: A Conjoint Study Examining Consumers' Willingness to Buy,” British Food Journal, Vol. 111, No. 7, 2009, pp. 660-668. doi:10.1108/00070700910972350

[60] D. Sylvester, K. Abbott and G. Marchant, "Not Again! Public Perception, Regulation, and Nanotechnology," Regulation and Governance, Vol. 3, No. 2, 2009, pp. 165-185. doi:10.1111/j.1748-5991.2009.01049.x

[61] K. Lyons, "Nanotechnology: Transforming Food and the Environment,” Food First Backgrounder, Vol. 16, No. 1, 2010, pp. 1-4.

[62] E. Court, S. Daar, E. Martin, T. Acharya and P. Singer, "Will Prince Charles et al. Diminish the Opportunities of Developing Countries in Nanotechnology?” 2004. http://nanotechweb.org/cws/article/indepth/18909

[63] http://www.publications.parliament.uk/pa/ld200910/ldsel ect/ldsctech/22/22i.pdf

[64] R. Harrington, "Nano Risk Assessment a Work in Progress," 2010.

http://www.foodnavigator.com/Science-Nutrition/Nano-ri sk-assessment-a-work-in-progress

[65] J. Falck-Zepeda, A. Cavialeri and P. Zambrano, "Delivering Genetically Engineered Crops to Poor Farmers: Recommendations for Improved Biosafety Regulations in Developing Countries,” IFPRI Brief 14, International Food Policy Research Institute, Washington DC, 2009. 